\title{
A cutaneous rash with mixed gell coombs allergic features, sclerodermoid changes and status post previous therapy
}

\author{
Ana Maria Abreu Velez ${ }^{1}$, Josepha Devaro², Michael S. Howard ${ }^{1}$ \\ ${ }^{1}$ Georgia Dermatopathology Associates, Atlanta, Georgia, USA, ${ }^{2}$ Coastal Dermatology, Savannah, Georgia, USA
}

Corresponding author: Ana Maria Abreu Velez, E-mail: abreuvelez@yahoo.com

\begin{abstract}
Allergies and autoimmune diseases may both be considered hyper-immune responses, where the body's immune system becomes supercharged and attacks or responds to inappropriate antigens. We describe a skin rash with a mixed allergic and autoimmune host response. A 65 year old female consulted her dermatologist for a pruritic rash. The patient had taken many medications without improvement of the rash, and lived in an area affected by environmental spills; other patients had presented with similar rashes concurrently. A clinical evaluation was performed, and skin biopsies were obtained for hematoxylin and eosin (H\&E) examination, as well as for immunohistochemical (IHC) and direct immunofluorescence (DIF) studies. The H\&E review revealed a mild, superficial, perivascular dermal infiltrate of lymphocytes, histiocytes, mast cells and eosinophils. Dermal sclerodermoid alterations were also noted. A mild peripheral blood eosinophilia was found; cutaneous IHC staining revealed staining for anti-HLA-DP, DQ, DR antigen and Complement/C5b-9/MAC, in the areas of the perivascular infiltrate and the sclerodermoid changes. The DIF confirmed these findings. Our case is characterized by a mixed allergic/autoimmune reaction, which did not fit exclusively into any single Gell Coombs immune response category.
\end{abstract}

Key words: Shotgun therapy; Skin rash; Gell coombs classification of allergic reactions; Clorox ${ }^{\circledR}$

\section{INTRODUCTION}

One of the major problems that health personnel face when evaluating skin rashes is prior "shotgun" therapy; specifically, often patients take multiple medications to address the initial rash, and thus are exposed to multiple putative allergens. Additionally, patients are often seen by several health providers that before the initial rash is properly diagnosed.

Evaluating skin rashes may also be difficult in areas subject to environmental toxins, especially when several patients in a limited geographic area present simultaneously with skin rashes. Under these conditions, initial diagnoses considered often include allergies (seasonal and/or due to specific antigens including pollen, dust or others) [1-4]. Contact dermatitis is also part of the initial differential diagnosis. Here we present a very unusual cutaneous rash case, with a combined immune response.

\section{CASE REPORT}

A 65 year old female resident of Savannah, Georgia visited the dermatologist with a history of six months of a recurrent, itchy rash manifested as papules on her arms, back, chest, and legs. The patient stated the lesions started after she cleaned several trailers, using strong detergents and Clorox ${ }^{\circledR}$. She denied constitutional symptoms, or gastrointestinal complaints. The patient owned multiple dogs, cats, horses, goats and a donkey. Prior to presentation, the patient had been previously seen by both immunologists and dermatologists for the rash, and had been evaluated for insect bites and/or skin infections; all previous evaluations were negative. The physical examination revealed papules on the patient's arms, back, chest and legs. Similar cases had been concurrently reported to local health authorities in the geographic area, and local environmental toxin contamination had been previously documented.

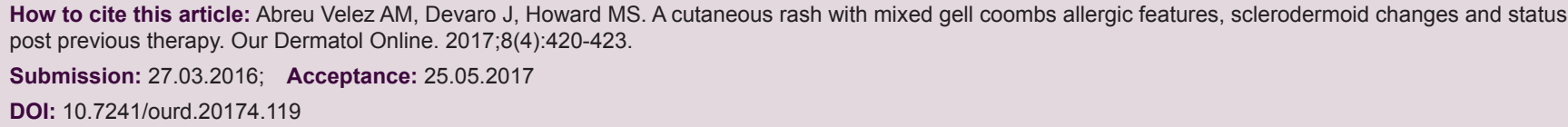


The patient received Pyrmethamine ${ }^{\circledR}$ cream, three separate bursts of oral steroids, Keflex, ${ }^{\circledR}$ Augmentin, ${ }^{\circledR}$ and Ivermectin tablets repeated for 10 days with no improvement. Most of her blood workup was normal electrolyte panel, hepatic function testing, BUN, creatinine, TSH, and ANAs). A complete blood count showed a mild eosinophila ( 9 with a normal range of 0-7). A Prednisone taper was given as well as Atarax, ${ }^{\circledR}$ Zyrtec $^{\circledR} 10 \mathrm{mgs}$, and Benadryl $25 \mathrm{mgs}$ at bed time. A RAST test was negative. Heavy metal panels for her blood and urine were also negative (testing for arsenic, cadmium, lead and mercury). Tissue transglutaminase (TTG) antibody, IgA and anti-IgA anti-endomysium antibodies were negative. Skin biopsies for hematoxylin and eosin (H\&E) staining, for immunohistochemistry (IHC) and for direct immunofluorescence (DIF) were taken.

No patient identifiers were recorded, and our research was conducted following medical guidelines of nondisclosure. Lesional skin was biopsied and studied utilizing hematoxylin and eosin (H\&E) staining, as well as via IHC, and direct immunofluorescence; all techniques were performed as previously described $[5,6]$.

\section{Microscopic Description}

Examination of the H\&E tissue sections demonstrated a histologically normal epidermis. Within the dermis, a mild, superficial, perivascular infiltrate of lymphocytes, histiocytes, mast cells and occasional eosinophils was seen. Dermal sclerodermoid alterations were also noted. A mild, superficial, perivascular dermatitis with an additional sclerodermoid component was diagnosed (Fig. 1).

\section{DIF}

The DIF examination revealed $\operatorname{IgG}(+++$, diffuse dermal matrix fibers); $\operatorname{IgA}, \operatorname{IgM}, \operatorname{IgD}, \operatorname{IgD}$ and $\operatorname{IgE}$ (+, focal within dermal hair shafts); Complement/ Clq $(++$, diffuse dermal matrix fibers, piloerector basement membrane and focal within hair follicles); Complement/C3 (focal spotty positivity at BMZ, and on neurovascular supplies into hair follicles); Kappa light chains $(++$, diffuse dermal matrix fibers in the central dermis); Lambda light chains $(++$, diffuse dermal matrix fibers and dermal nerves); albumin $(++$, dermal perivascular) and fibrinogen $(++$, diffuse dermal matrix fibers, focal dermal perivascular, on piloerector muscles and on dermal nerves) (Fig. 1).

\section{IHC}

Our IHC staining revealed positive strong staining with anti-HLA DP, DQ, DR antigen throughout the neurovascular dermal plexus, as well as on vessels around eccrine sweat glands (Fig. 1). Complement/ C5b-9/MAC was positive around hair follicles and sebaceous glands, throughout the neurovascular plexus and in the areas of sclerodermoid changes seen by H\&E review. CD45 positive cells were seen around the upper and middle dermal blood vessels, and around vessels supplying eccrine gland ducts and neurovascular packages in the dermis (Fig. 1) Staining for CD3 and CD8 was mildly positive around the superficial dermal blood vessels. Dermal staining for CD20 was negative. Factor XIIIa staining was positive around the sclerodermoid areas, and around dermal blood vessels. Staining for CD68 was positive around dermal blood vessels, piloerector muscles, and in the sclerodermoid areas (Fig. 1). Staining for CDla was positive in the areas of the dermis below the inflamed vessels.

\section{DISCUSSION}

We present a case of a skin rash with sclerodermoid changes, and an allergic-like immune response in a geographic area where similar, concurrent cases were seen in other patients. An eosinophilia-myalgia syndrome secondary to L-tryptophan-containing products or toxic oils was considered in the differential diagnosis [7]. The serum and skin biopsy eosinophilia, and the IgE detected by DIF indicated an allergic component. However, the dermal sclerodermoid alterations, combined with the positive HLA-DR, DP, DQ antigen and Complement/C5b-9/MAC IHC staining suggested antigen presentation via a likely environmental trigger component. Heavy metal testing displayed negative results. The significant exposure to Clorox may represent a primary sensitizing factor in the case.

The RAST tests currently on the market do not contain many of the allergens a patient may be exposed to. Our patient had significant contact with Clorox, ${ }^{\circledR}$ and animals; she was also likely exposed to environmental toxins, given her geographic location. Often environmental spills are not reported to the authorities; the observed neural reactivity and sclerodermoid changes in the skin suggest a possible toxin exposure. 


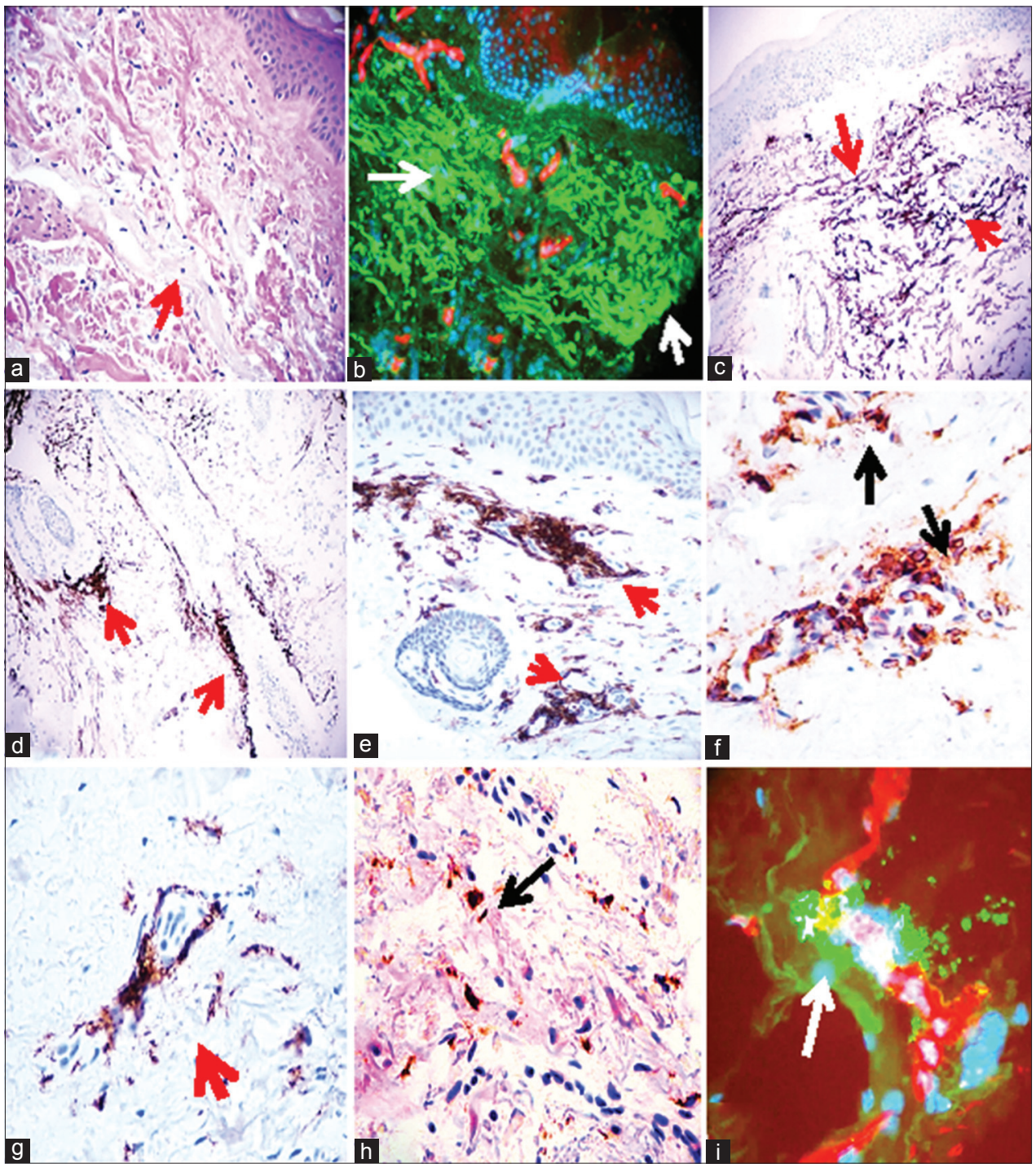

Figure 1: (a) H\&E staining demonstrates dermal edema and solar damage (100X, red arrow). (b) DIF using FITC conjugated IgG antibody, and showing positive staining on dermal collagen fiber (green staining; white arrows) (100X). Cell nuclei are counterstained with DAPI in blue; the red staining represents Texas red conjugated Ulex antibody on dermal blood vessels. (c) IHC staining shows positive Complement/C5b-9 stain (brown staining; red arrows), in the same areas of the abnormal dermal fibers seen by H\&E. In (d) IHC staining for Complement/C5b-9 against the hair follicles and sebaceous glands, and in (e) against the vessel s(brown staining; red arrows). (f) Double IHC staining showing positive HLA-DR, DP, DQ antigen staining (brown) colocalizing with von Willembrand factor staining (red), around the upper dermal inflamed vessels (black arrows) (400X). (g) IHC staining showing positive CD45 staining around dermal blood vessels and nerves (brown staining; red arrow). (h) IHC double staining using CD68 (brown staining; black arrow) and Factor XIIla (pink/purple), showing positivity to the vessels and also to extracellular structures. (i) DIF using FITC conjugated anti-human IgD antibody showing positive staining on dermal neurovascular structures (green staining; white arrow).

The histopathologic and immunodermatologic findings of this patient did not fit exclusively into a single diagnostic category (e.g., contact dermatitis, allergic dermatitis, drug eruption), and sclerodermoid changes were also noted. In similar cases, we recommend evaluating the patient for possible heavy metal and toxins in blood, hair and/or nail tissue, especially when other people in the patient's community are presenting with similar clinical findings. Complement/C5b-9 IHC staining was positive in several areas of the skin, and could be detected in early and late stages of some sclerodermoid conditions triggered by organic solvents, silica or other agents (and not detected via heavy metal panels or RAST testing) [8,9].

In complex rash cases, another complicating diagnostic factor is previous treatment by multiple healthcare providers. Often, multiple medications are prescribed when a causative diagnosis is not established; the medications then may complicate discovery of the true cause of the disease. We recommend a complete clinical examination before initiation of any therapy, prescribing antihistamines to address pruritus while working to achieve an accurate diagnosis. 


\section{ACKNOWLEDGEMENT}

Jonathan S. Jones, HT (ASCP) at Georgia Dermatopathology Associates provided excellent technical assistance.

\section{Abbreviations and Acronyms}

Immunohistochemistry (IHC), direct and indirect immunofluorescence (DIF, IIF), hematoxylin and eosin (H\&E), basement membrane zone (BMZ), fluorescein isothiocyanate (FITC), 4',6-diamidino-2-phenylindole (DAPI).

\section{REFERENCES}

1. Edele F, Esser PR, Lass C, Laszczyk MN, Oswald E, Strüh CM, et al. Innate and adaptive immune responses in allergic contact dermatitis and autoimmune skin diseases. Inflamm Allergy Drug Targets. 2007;64:236-44.

2. Merk HF, Sachs B, Baron J. The skin: target organ in immunotoxicology of small-molecular-weight compounds. Skin Pharmacol Appl Skin Physiol. 2001;14:419-30.

3. Kallinich T. Regulating against the dysregulation: new treatment options in autoinflammation. Semin Immunopathol. 2015;37:429-37.
4. Coombs RRA, Gell PGH. Classification of allergic reactions responsible for clinical hypersensitivity and disease. In: Clinical Aspects of Immunology, 3rd edn. (ed. by P.G.H. Gell, R.R.A. Coombs, P.J. Lachmann), 1975 pp. 761-781. Blackwell Scientific Publications, London.

5. Abreu Velez AM, Smoller BR, Howard MS. Rouleaux and autoagglutination of erythrocytes associated with fibrin-like material in skin biopsies form patients with autoimmune blistering diseases. Our Dermatol Online. 2013;4(Suppl.3):613-5.

6. Abreu-Velez AM, Brown VM, Howard MS. A transient drug induced lupus erythematosus - like allergic drug reaction with multiple antibodies. Our Dermatol Online. 2013;4:511-3.

7. CDC. Eosinophilia-myalgia syndrome and L-tryptophan-containing products--New Mexico, Minnesota, Oregon, and New York, 1989. MMWR 1989;38:785-8.

8. Garcia-Zamalloa AM, Ojeda E, Gonzales-Beneitez C, Goni J, Garrido A. Systemic sclerosis and organic solvents: early diagnosis in industry. Ann Rheum Dis. 1994;53:618.

9. de la Espriella J, Crickx B. [Scleroderma and environment. I. Scleroderma and sclerodermiform states induced by silica and chemical agents or drugs]. Ann Dermatol Venereol. 1991;118:948-53.

Copyright by Ana Maria Abreu Velez, et al. This is an open-access article distributed under the terms of the Creative Commons Attribution License, which permits unrestricted use, distribution, and reproduction in any medium, provided the original author and source are credited.

Source of Support: Georgia Dermatopathology Associates, Atlanta, Georgia, USA, , Conflict of Interest: None declared. 